\title{
Single-Photon Source for Quantum Information Based on Single Dye Molecule Fluorescence in Liquid Crystal Host
}

\author{
Svetlana G. Lukishova \\ Russell P. Knox \\ Patrick Freivald \\ Andrew McNamara \\ Robert W. Boyd \\ Carlos R. Stroud, Jr.
}

The Institute of Optics, University of Rochester, Rochester,

New York, USA

\section{Ansgar W. Schmid \\ Kenneth L. Marshall}

Laboratory for Laser Energetics, University of Rochester, Rochester, New York, USA

This paper describes a new application for liquid crystals: quantum information technology. A deterministically polarized single-photon source that efficiently produces photons exhibiting antibunching is a pivotal hardware element in absolutely secure quantum communication. Planar-aligned nematic liquid crystal hosts deterministically align the single dye molecules which produce deterministically polarized single (antibunched) photons. In addition, 1-D photonic bandgap cholesteric liquid crystals will increase single-photon source efficiency. The experiments and challenges in the observation of deterministically polarized fluorescence from single dye molecules in planar-aligned glassy nematic-liquid-crystal oligomer as

The authors acknowledge the support by the U.S. Army Research Office under Award No. DAAD19-02-1-0285 and National Science Foundation Awards ECS-0420888, EEC0243779, PHY-0242483. The work was also supported by the U.S. Department of Energy Office of Inertial Confinement Fusion under Cooperative Agreement No. DE-FC0392SF19460, the University of Rochester, and the New York State Energy Research and Development Authority. The support of DOE does not constitute an endorsement by DOE of the views expressed in this article.

Receipt of LC oligomers from S.-H. Chen, Cornerstone Research Group, Inc. and F. Kreuzer of Wacker, Munich are gratefully acknowledged. The authors thank L. Novotny, A. Lieb, J. Shojaie, A. Trajkovska, S. Culligan, for advice and help, and J. Starowitz for support in optical material laboratory.

Address correspondence to Svetlana G. Lukishova, The Institute of Optics, University of Rochester, Rochester, New York, 14627, USA. E-mail: sluk@lle.rochester.edu 
well as photon antibunching in glassy cholesteric oligomer are described for the first time.

Keywords: antibunching; liquid crystals; single-dye molecules; single-photon source

\section{INTRODUCTION}

Quantum information in the form of quantum communication and quantum computing [1-3] is currently an exceedingly active field. A single-photon source (SPS) that efficiently produces photons with antibunching characteristics [4] is one of the pivotal hardware elements for photonic quantum information technology. For single photons, the second order correlation function $g^{(2)}(\tau)=\langle I(t) I(t+\tau)\rangle /\langle I(t)\rangle^{2}$ should have a minimum at $\tau=0$ (in an ideal case $g^{(2)}(0)=0$ ), indicating the absence of pairs, i.e., antibunching [5]. Here $I$ is intensity.

Using a SPS, secure quantum communication will prevent any potential eavesdropper from intercepting a message without the receiver noticing [6]. In another implementation, a SPS becomes the key hardware element for quantum computers with linear optical elements and photodetectors [7]. Because of the difficulties in developing robust and efficient sources of single photons, current quantum communication systems which are already marketed [8], are using the ordinary, low-efficient photon sources attenuated to the single-photon level ( $\sim 0.1$ photon per pulse on average). In addition, the attenuated photon sources are contaminated with double and triple photons. An efficient (with an-order-of-magnitude-higher photon number per pulse) and reliable light source that delivers a train of pulses containing each one, and only one, photon is a very timely challenge. Another important property of the ideal SPS connected with its efficiency is the deterministic polarization of single photons as the transferred information is coded in the polarization states.

The critical issue in producing single photons by a method other than by trivial attenuation of a beam is the very low concentration of photon emitters dispersed in a host, such that, within an excitation-laser focal spot, only one emitter becomes excited (see Figure 1) which will emit only one photon at a time, because of its finite fluorescence lifetime. Most single-photon sources developed heretofore, e.g., based on heterostructured quantum dots [9], operate only at liquid He temperature. In addition, they are of low efficiency, not polarized deterministically, and not readily tunable. To date, three approaches have been suggested for room-temperature single-photon sources based on fluorescence from single dye molecules [10], colloidal 


\section{Laser beam}

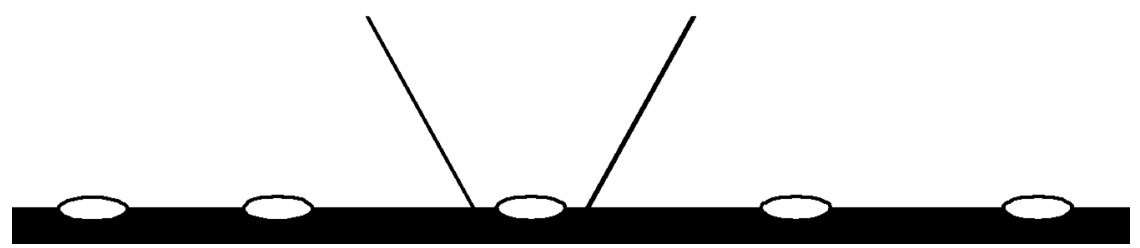

FIGURE 1 Schematic of single-molecule excitation.

semiconductor quantum dots (nanocrystals) [11], and color centers in diamond [12]. In addition to the above mentioned disadvantages, the color center source suffers from the problem that it is not easy to outcouple the photons, and that the spectral spread of the light is typically quite large $(\sim 120 \mathrm{~nm})$. Both single molecules and colloidal quantum dots dissolved in a proper solvent can be embedded in planar aligned liquid crystals (LCs) [13-15] to circumvent the deficiencies that plague the other systems. Single atoms coupled to high-finesse cavities have achieved very impressive couplings of $40-70 \%$, but they have other formidable problems [16].

The main challenge of SPS based on dye-fluorescence is dye bleaching. A few years ago, major progress towards overcoming this challenge was achieved: single terrylene molecules did not bleach in a paraterphenyl microcrystal host for several hours of continued, pulsed laser excitation [17]. The paraterphenyl host protected the dye molecules from the oxygen causing their bleaching. Recently we succeeded in avoiding dye-bleaching as well, driving specially treated dye-doped LC hosts for more than one hour by $c w$-laser excitation [13-15].

In the present paper, we demonstrate for the first time deterministically polarized fluorescence from single dye molecules. Planaraligned, nematic LC hosts provide deterministic alignment of single dye molecules in a preferred direction. The structure of this paper is as follows. Section 2 describes glassy-oligomer-LC sample preparation and the alignment procedure for single-molecule fluorescence microscopy. Section 3 describes the experimental setup for singlemolecule fluorescence microscopy and photon antibunching correlation measurements. In Section 4, part 1, the results on deterministically polarized fluorescence of single dye molecules in planar-aligned glassy LC oligomers are reported, including the challenges of single-molecule fluorescence microscopy of aligned LCs. In part 2 of Section 4, we present the results of photon antibunching in glassy cholesteric LC host. Section 5 concludes the paper. 


\section{PREPARATION AND CHARACTERIZATION OF LIQUID CRYSTAL SAMPLES DOPED WITH SINGLE DYE MOLECULES}

\subsection{Preparation of Liquid Crystals Doped with Single-Molecules of Fluorescent Dyes}

To focus the laser beam on the single emitter, e.g., on the fluorescent dye molecule, the LC should be doped with dye at an extremely low concentration, such that the final sample contains only a few molecules per $\mu \mathrm{m}^{2}$ irradiation area (see Fig. 1).

Single-molecule fluorescence microscopy imposes a requirement on the sample thickness: $\sim 180$ and $300 \mu \mathrm{m}$-working-distance, high-N.A. objectives permit use only of samples with thickness not exceeding this value. For this reason, $\sim 170-\mu \mathrm{m}$-thickness glass microscopic cover slip substrates (Corning) were used that both are fragile and need special care in handling.

To minimize false fluorescence contributions by contaminants during single-molecule-fluorescence microscopy, rigorous cleaning of glass slips is mandatory [18]. Ordinary substrate cleaning procedures for LC displays are not sufficient. LC cells are fabricated in a class 10,000 LC clean-room facility. Ultrasonic cleaning for 60 minutes frees the $1^{\prime \prime} \times 1^{\prime \prime}$ slips from any dirt particles. Slips are then rinsed in flowing, deionized water, dried in a stream of compressed nitrogen, and washed in toluene from organic components. To remove the toluene, slips are washed again with pure ethanol and dried. After that, they are being etched in piranha solution $\left(\mathrm{H}_{2} \mathrm{SO}_{4}+\mathrm{H}_{2} \mathrm{O}_{2}\right.$ in equal volume concentration) for about 20 minutes, rinsed in flowing, deionized water and dried in a stream of oil-free nitrogen.

Proper dye concentration in the solution for single-molecule fluorescence microscopy was established by iterative trial and error (ordinarily of the order of $10^{-8}-10^{-10} \mathrm{M}$ ). In sequential dilution steps of dye in solvent (chlorobenzene or methylene chloride), solutions were spun onto glass slips, and for each concentration, confocal fluorescence microscopy determined by the count rate the final emitter concentration per irradiation volume. Once single molecules were predominantly observed, the dilution endpoint was reached. This final dye solution was mixed with powder of the LC oligomer (1-8\% weight concentration of oligomer). At the final stage, the oligomer/dye solution was filtered through $0.2-0.45 \mu \mathrm{m}$ particle filters.

We used two types of glassy-LC oligomers with low fluorescence background for both nematic and cholesteric in powder form: (1) synthesized by S.H. Chen's group (University of Rochester) [19], and 

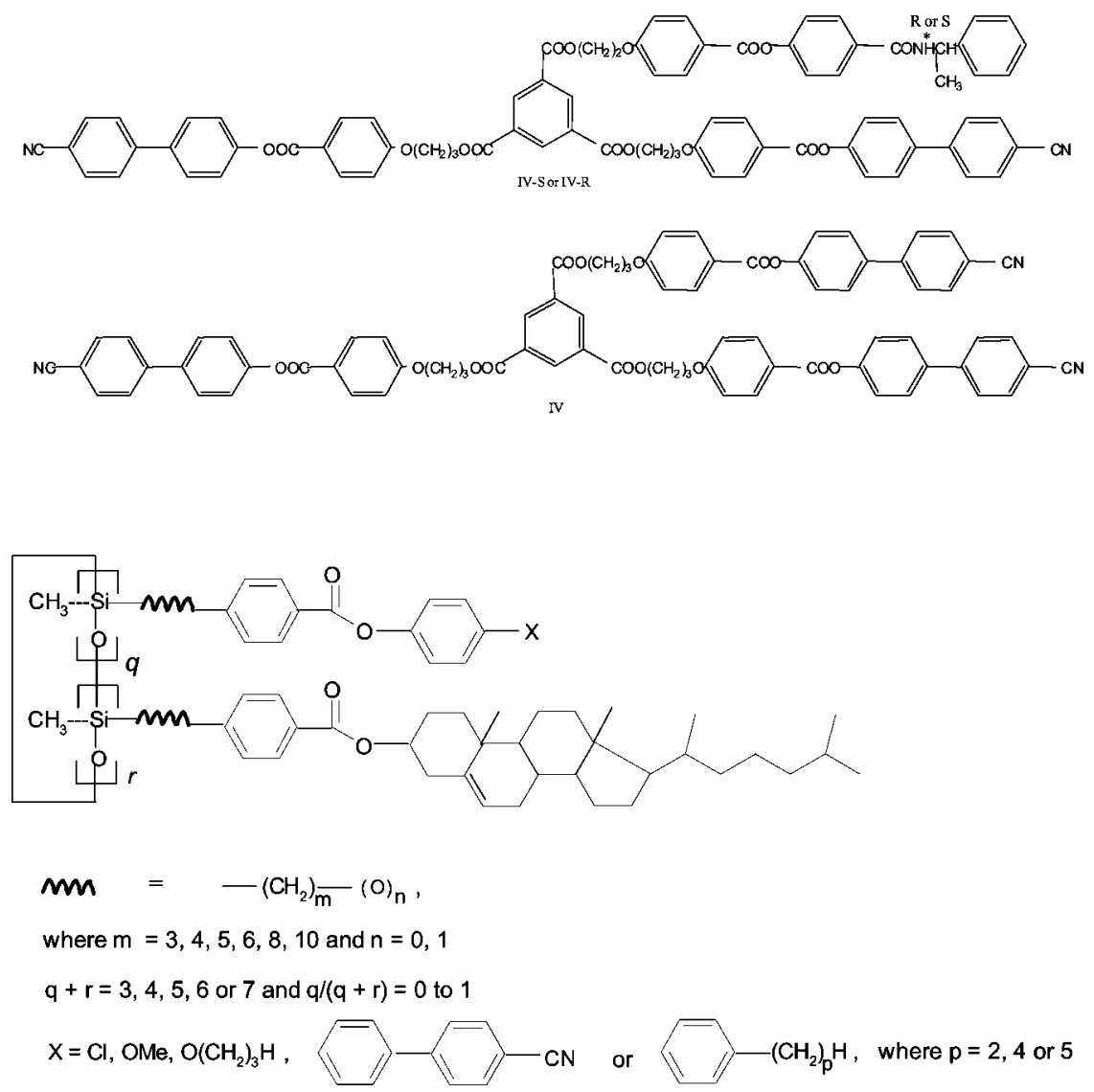

FIGURE 2 Molecular structure of Chen's glassy LC oligomers (top) and Wacker LC oligomers (bottom).

(2) purified Wacker oligomers [20]. The molecular structure of both types of oligomers is shown in Figure 2. Some oligomers with molecular structure of Figure 2, top were synthesized by Cornerstone Research Group, Inc. Both types of used oligomers have LC ordering at elevated temperatures. The nematic or cholesteric LC state of these materials was preserved at room temperature by slow cooling to the glassy state with frozen nematic/cholesteric order.

The following fluorescent dyes were used in our experiments: $\operatorname{DiIC}_{18}(3)$ from Molecular Probes and terrylene synthesized by W. Schmidt [21]. The molecular structures of these dyes are presented in Figure 3. The fluorescence maximum of both dyes lies near $580 \mathrm{~nm}$. 


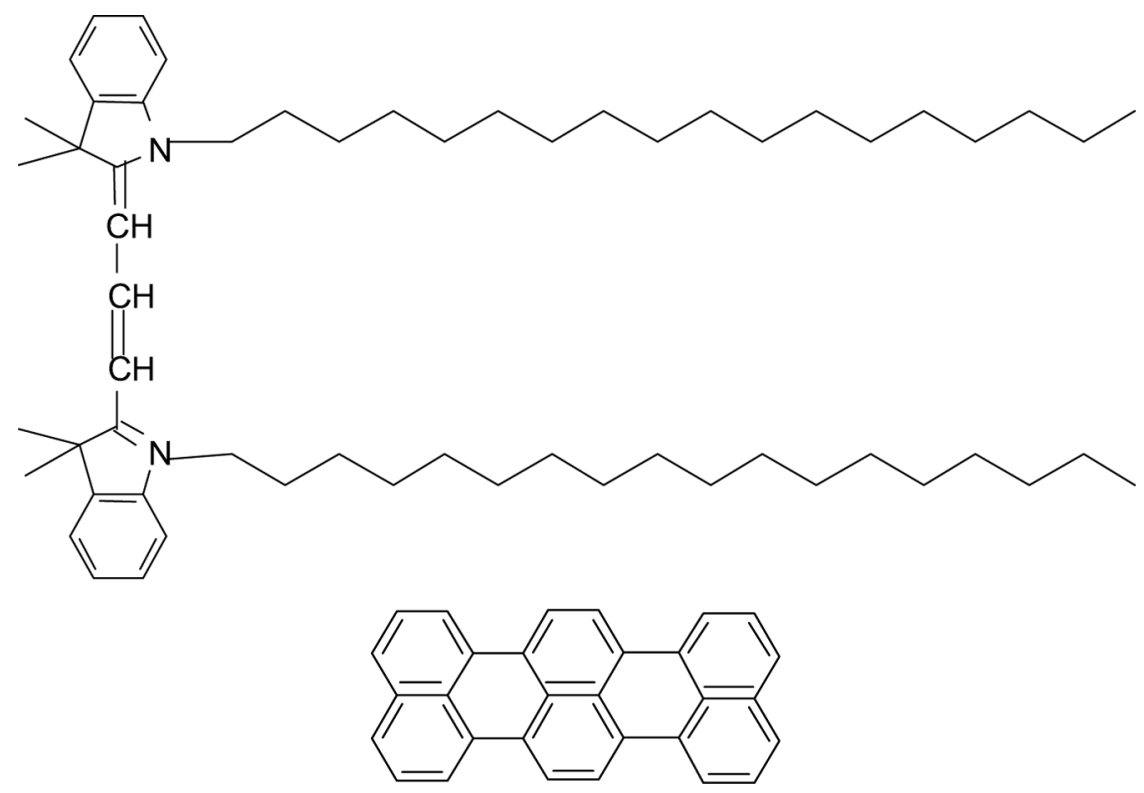

FIGURE 3 Molecular structures of $\operatorname{DiIC}_{18}(3)$ (top) and terrylene (bottom) dyes.

\subsection{Alignment of Liquid Crystals for Single Molecule Fluorescence Microscopy}

Three different planar alignment procedures for LCs were used: (i) substrate shearing, (ii) buffing, and (iii) photoalignment. For sheared samples, no additional substrate coatings were needed. For buffing, substrates were spin coated with either of two polymers: Nylon $6 / 6$ or Polyimid. For buffing, we used a standard, velvet-surface buffing machine.

To prevent damage of the fragile slips during the buffing procedure, they were "blocked to" 1-mm-thick microscope slides with watersoluble acetate, using 40 -min heating at $80^{\circ} \mathrm{C}$ for better results. After buffing, the slips were unblocked in standing, deionized water over night. This was followed by a rinse in flowing, deionized water to rid the samples of acetate traces.

For photoalignment, slips were spin-coated with Staralign. Photoalignment of coated polymer was achieved using six, UV discharge lamps RPR 3000 (Southern New England Ultraviolet Co.) with maximum wavelength $\sim 302 \mathrm{~nm}(40 \mathrm{~nm}$ bandwidth) and a UV linear dichroic polarizer (Oriel), $1.74^{\prime \prime} \times 1.74^{\prime \prime}$ large, placed in a hermetic box. The photoalignment procedure at $\sim 5 \mathrm{~mW} / \mathrm{cm}^{2}$ power density at $302 \mathrm{~nm}$ lasted 10 minutes. We used a $75-\mathrm{W}$, Xenon light source 

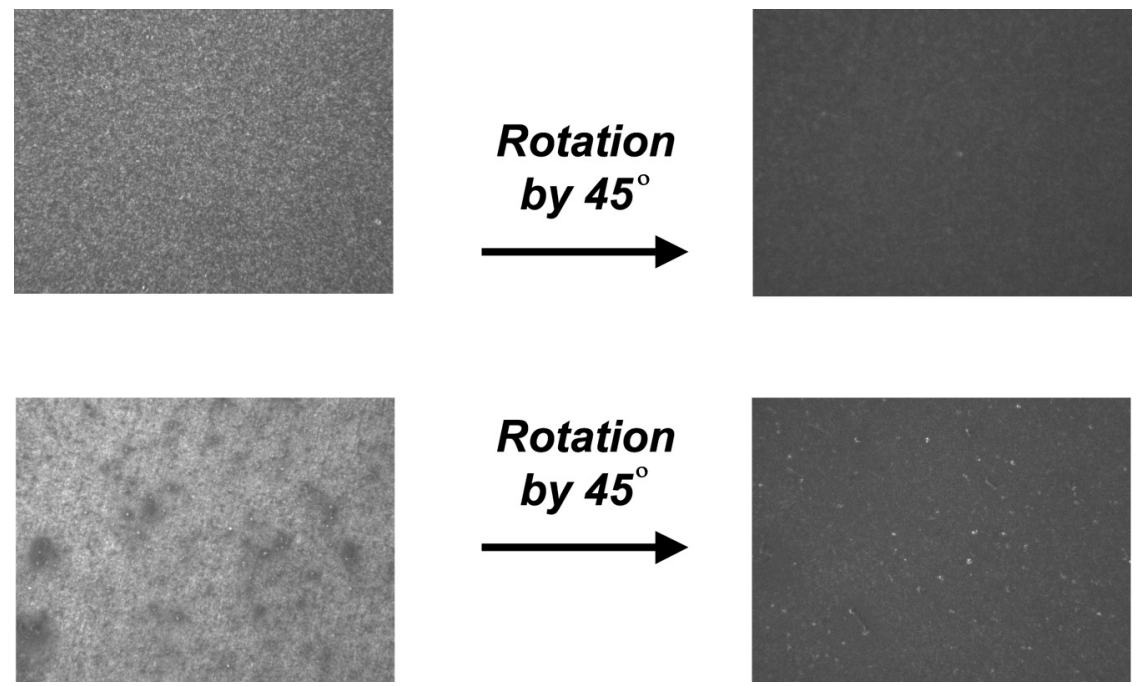

FIGURE 4 Polarizing microscope images of photoaligned (top) and buffed (bottom) planar-aligned glassy nematic oligomer LC films $(0.6 \mathrm{~mm} \times 1.2 \mathrm{~mm}$ area): left images are between cross polarizers showing uniform birefringence; right images - samples are rotated by $45^{\circ}$ showing a significant darkening of the field (evidence of good planar alignment).

(Photon Technology International, Inc.) as well with a stack of linear polarizing quartz plates. This new alignment technique excludes contamination of the material by particulates.

We prepared $\sim 100$-nm-thickness films of Chen's glassy nematic LCs doped with dye molecules aligned deterministically both through photoalignment and buffing. In the case of LC oligomers which are solid at room temperature, a strict annealing regime is needed for a good-quality alignment to prevent destruction of the film during heating/cooling process. We succeeded in the preparation of such alignment (Fig. 4) on an area at least $10 \mathrm{~mm} \times 10 \mathrm{~mm}$ using both photoalignment (Fig. 4, top) and buffing (Fig. 4, bottom). Dye-doped Wacker oligomer alignment was made by shearing the substrates in the heated state and slowly cooling them to room temperature.

\section{EXPERIMENTAL SETUP}

Single-molecule fluorescence microscopy was carried out on Witec alpha-SNOM device in confocal transmission mode. Figure 5 shows the schematics of this experiment. The dye-doped LC sample was placed in the focal plane of a $0.8-$ N.A. or 1.4-N.A. oil-immersion 

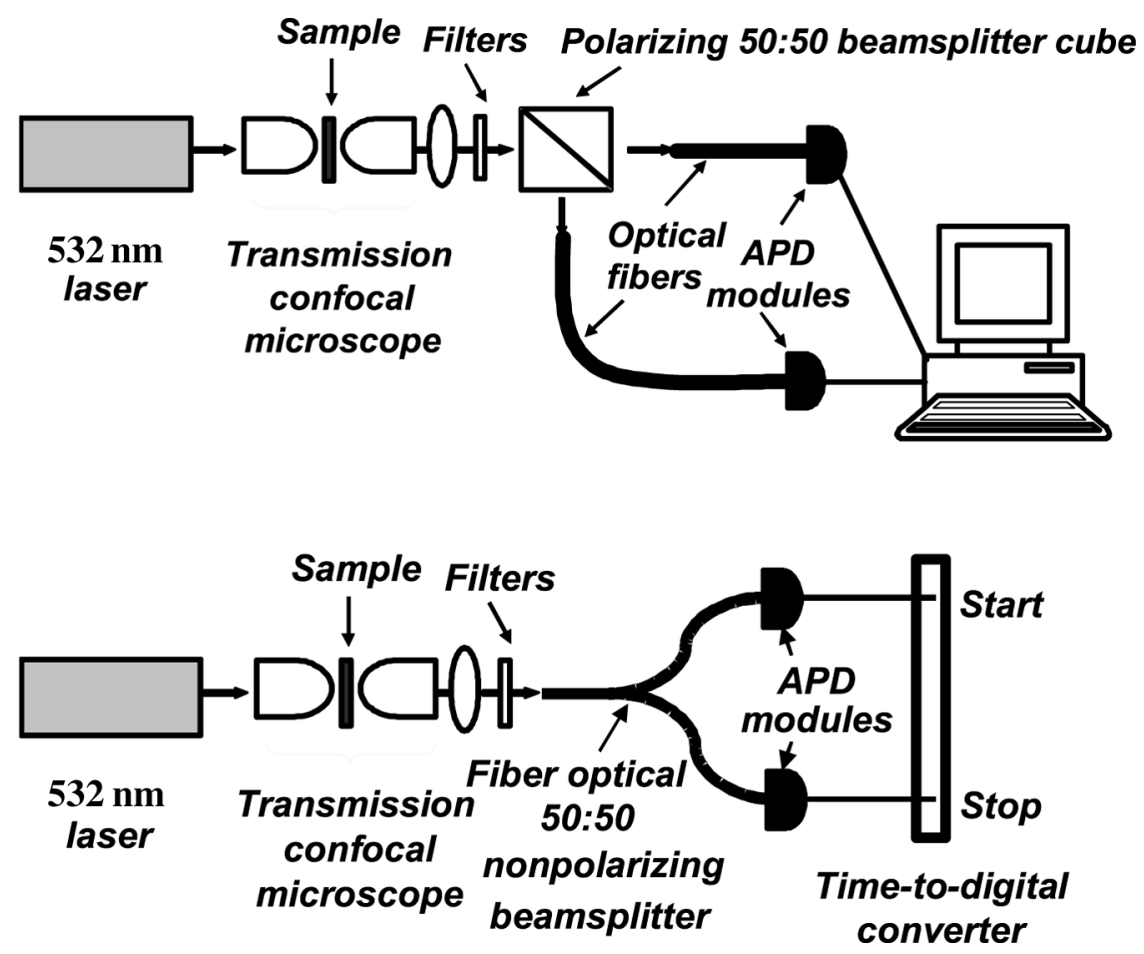

FIGURE 5 Experimental setup for deterministically polarized fluorescence (top) and for antibunching correlation measurements (bottom).

microscope objective. The sample was attached to a piezoelectric, XYZ translation stage. Light emitted by the sample was collected by a confocal setup using a 1.25-N.A., oil-immersion objective together with an aperture in form of a 50-100- $\mu \mathrm{m}$-core optical fiber. The $\mathrm{cw}$, spatially filtered (through a single-mode fiber), linearly polarized (contrast $10^{5}: 1$ ), 532-nm diode-pumped Nd:YAG laser output excited single molecules. In focus, the intensities used were of the order of several $\mathrm{kW} / \mathrm{cm}^{2}$.

For polarized fluorescence measurements we used a 50/50 polarizing beamsplitter cube with the confocal microscope apertures in form of 50-100- $\mu \mathrm{m}$-core optical fiber placed in each arm at beamsplitter output (Fig. 5, top). In the case of antibunching correlation measurements, the collection fiber was part of a non-polarization-sensitive $50 / 50$ fiber splitter that formed the two arms of a Hanbury Brown and Twiss correlation setup [22] (Fig. 5, bottom). Residual, transmitted excitation light was removed by two consecutive dielectric interference filters or by a combination of a notch filter and Schott 
orange-glass filter yielding a combined rejection of better than seven orders of magnitude at $532 \mathrm{~nm}$.

Photons in the two arms were detected by identical, cooled avalanche photodiode modules (APD) in single-photon-counting, Geiger mode (Perkin Elmer SPCM AQR-14). The time interval between two consecutively detected photons $n(\tau)$ in separate arms were measured by a 68-ns-full-scale Phillips Scientific-7186 time-to-digital converter using a conventional start-stop protocol. Within this converter's linear range, the time uncertainty in each channel corresponds to $25 \mathrm{ps}$. A PC stored $n(\tau)$, the number of counts per defined time interval $\tau$. It has been proved experimentally (see, e.g., Refs. [23] and [24]) that a very good approximation of $g^{(2)}(\tau)$ comes directly from the coincident events $n(\tau)$, for relatively low detection efficiency and therefore low counting rate. That is why we consider $n(\tau)$ to be proportional to the autocorrelation function $g^{(2)}(\tau)$.

\section{EXPERIMENTAL RESULTS}

\subsection{Deterministically Polarized Single-Molecule Fluorescence from Doped Glassy Nematic Oligomers}

For these experiments we used $\operatorname{DiIC}_{18}(3)$ dye (Fig. 3, top) in planaraligned, glassy nematic- LC hosts (Fig. 2, top IV) by Chen. Film thickness was $\sim 100 \mathrm{~nm}$ (Fig. 4, top).

Figure 6 shows images of single-molecule fluorescence [25] for components perpendicular (left) and parallel (right) to the alignment direction, under 532-nm, cw-excitation. These two polarization components in the plane of the sample have been separated with a polarizing beamsplitter cube (Fig. 5, top). Figure 6 clearly shows that for this sample, the polarization direction of the fluorescence of single molecules is predominantly in the direction perpendicular to the alignment of LC molecules. It is important that the background level of left and right images of Figure 6 is the same $(\sim 10$ counts/pixel or $\sim 640$ counts/s). Single-molecule fluorescence signal exceeds background by up to 15 times. The polarization anisotropy is defined here as $\rho=\left(\mathrm{I}_{\text {par }}-\mathrm{I}_{\text {perp }}\right) /\left(\mathrm{I}_{\text {par }}+\mathrm{I}_{\text {perp }}\right)$, where $\mathrm{I}_{\text {par }}$ and $\mathrm{I}_{\text {perp }}$ are fluorescence intensities for polarization components parallel and perpendicular to the alignment direction [26]. Processing Figure 6 images shows that from a total of 38 molecules, 31 molecules have a negative value of $\rho$. The same sign of the polarization anisotropy was obtained in spectrofluorimeter measurements for a sample with high $(\sim 0.5 \%$ by weight) concentration of the same dye in a planar aligned glassy nematic LC film with $\sim 4.1 \mu \mathrm{m}$ thickness. 


\section{$10 \mu \mathrm{m}$}

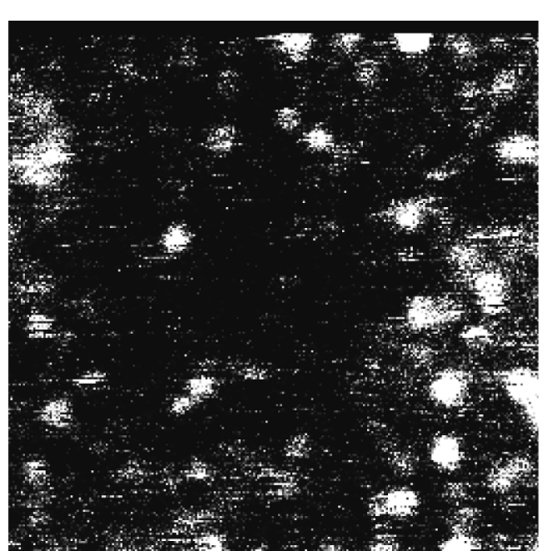

$10 \mu \mathrm{m}$

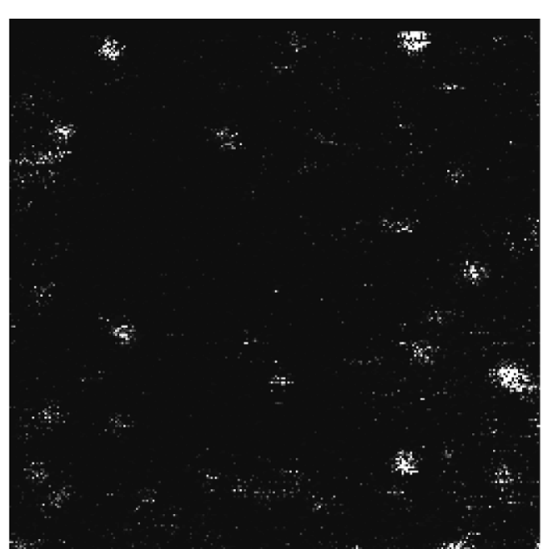

FIGURE 6 Confocal-fluorescence-microscopy images of $\mathrm{DiIC}_{18}(3)$ singlemolecule fluorescence in planar aligned glassy nematic LC host: left - polarization perpendicular to the alignment direction; right - parallel polarization.

This predominance of "perpendicular" polarization can be explained by $\operatorname{DiIC}_{18}(3)$ 's molecular structure (Fig. 3, top). The two alkyl chains would likely orient themselves parallel to the rod-like LC molecules, but the emitting/absorbing dipoles which are nearly parallel to the bridge (perpendicular to alkyl chains) will be directed perpendicular to the LC alignment. $\mathrm{DiIC}_{18}(3)$ molecules orient in the same manner in cell membranes [27,28].

Note, that the images in Figure 6 and all other single-molecule fluorescence images presented here were taken by raster scanning the sample relative to the stationary, focused laser beam. The scan direction was from left to right and line by line from top to bottom. The size of the bright features is defined by the point-spread function of the focused laser beam. These images contain information not only about the spatial position of the fluorescent molecules, but also about changes of their fluorescence in time. Dark horizontal stripes and bright semicircles instead of circles represent blinking and bleaching of the molecules in time. Blinking and bleaching are a common, singlemolecule phenomenon and convincing evidence of the single-photon nature of the source. The explanation of the nature of the long-time blinking from milliseconds to several seconds remains a subject of debates in the literature, see, e.g., Ref. [18].

The maximum count rate of single-molecule images was approximately $10 \mathrm{kcounts} / \mathrm{s}(\sim 160$ counts $/$ pixel with $\sim 4 \mathrm{~s}$ per line scan, 256 


\section{$10 \mu \mathrm{m}$}

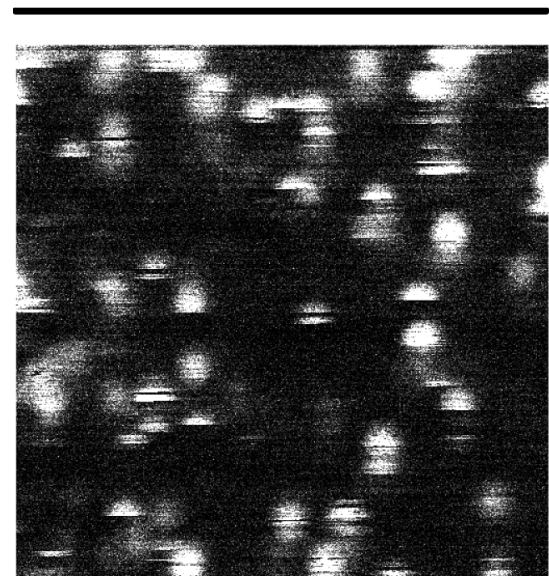

$10 \mu \mathrm{m}$

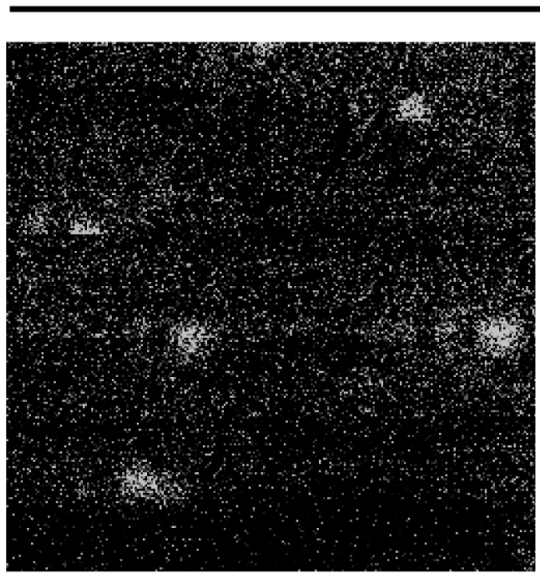

FIGURE 7 Parasitic fluorescence from single-molecule impurities in the photoalignment agent Staralign without UV-treatment (left) and undoped oligomer material (right).

pixels per line) with a fluorescence life time of the molecules approximately several ns. Note that the detector dark counts were fewer than 100 counts $/ \mathrm{s}$.

Seven molecules in Figure 6 have either positive anisotropy or equal zero. These molecules can be either a small amount of impurities in Staralign photoalignment agent which have not been bleached even after UV-irradiation of Staralign-coated slips, or impurities of the glassy oligomer host. Figure 7, left, shows single-molecule fluorescence images of photoalignment agent Staralign before the UV-irradiation. Single-molecule fluorescence microscopy method is very sensitive to the material impurities. Sometimes we observed single-molecule fluorescence from the impurities in glassy LC oligomers (Fig. 7, right) even when a chromatographic analysis did not show them. The thin films of Polyimid and Nylon 6/6 used for buffing alignment possess higher fluorescence count rate than single molecules of fluorescent dyes used in our experiments.

\subsection{Fluorescence Antibunching in Glassy Cholesteric Liquid Crystal Oligomers}

For these experiments Wacker glassy cholesteric LC oligomer (Fig. 2, bottom) doped with single molecules of terrylene dye (Fig. 3, bottom) 


\section{$10 \mu \mathrm{m}$}
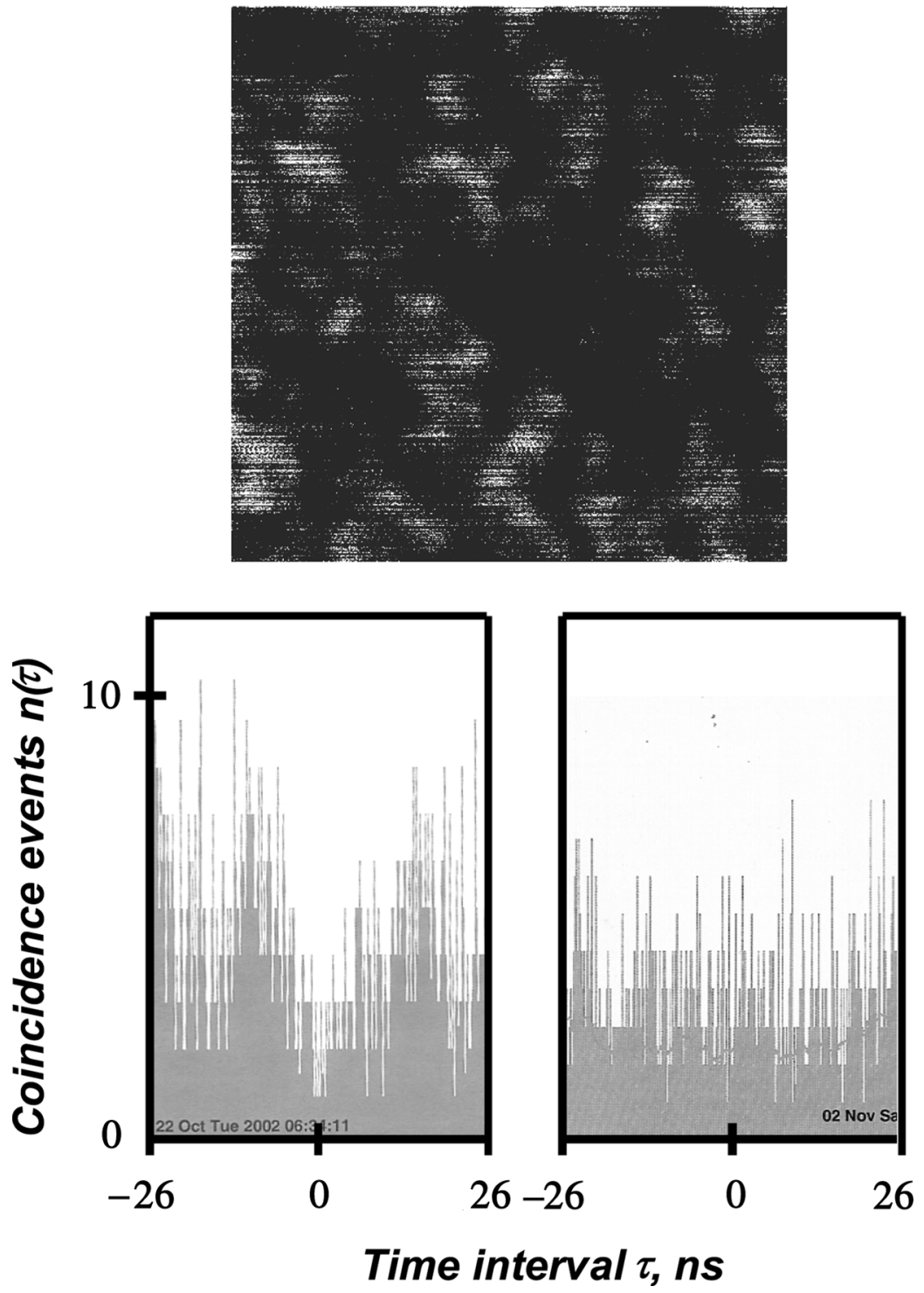

FIGURE 8 Confocal-fluorescence-microscopy image of single-terrylenemolecule fluorescence in planar aligned glassy Wacker oligomer cholesteric LC host (top) and the histograms of coincidence events $n(\tau)$ of the singleterrylene-molecule-fluorescence in glassy Wacker oligomer cholesteric LC host (bottom left) and of an assembly of several uncorrelated molecules (bottom right). Left histogram exhibits a dip at $\tau=0$ indicating photon antibunching. 
was used. Owing to fluorescent impurities, Wacker LCs as received had a high-fluorescence background in our experiments. We used purified Wacker oligomer with a $2.2 \mu \mathrm{m}$-selective reflection wavelength.

Figure 8, top, shows single terrylene-dye-molecule fluorescence obtained by confocal fluorescence microscopy. The scan speed was $\sim 3 \mathrm{~s}$ per line (512 pixels). A coincidence-count histogram $n(\tau)$ from single terrylene molecules in Wacker host exhibits a dip at $\tau=0$ (Left side of Fig. 8, bottom). The measured signal-to-background ratio of our experiments ranges from 2 to 8 , so the probability that a photon from the background triggers a coincidence with a photon from the molecule is very low. Because $n(\tau)$ is proportional to the autocorrelation function $g^{(2)}(\tau), n(0) \sim 0$ means that $g^{(2)}(0) \sim 0$ in our experiments. Two fluorescence photons are not observed within an arbitrarily short time interval. This fluorescence antibunching is due to the finite radiative lifetime of the molecular dipole and is therefore clear proof that we observed the emission of one, and only one, molecule. The histogram on the right from multiple, uncorrelated molecules in the same host shows no such dip at $\tau=0$, i.e., no antibunching.

\section{CONCLUSION}

This paper demonstrates for the first time, deterministically polarized fluorescence from single emitters at room temperature and fluorescence antibunching in an LC host. The challenges of single-molecule fluorescence microscopy of aligned glassy LC oligomers are described as well, especially background fluorescence from both the LC host and the alignment agents.

Our current SPS performance does not yet use all the capabilities offered by the LC host to increase the excitation and collection efficiency. Our estimations [13,14] show that an SPS efficiency increase of up to one order of magnitude can be expected, using photonic bandgap matching with the dye fluorescence band.

We plan to produce these tunable, room-temperature devices with an increase in output coupling efficiency, a narrowing of the fluorescence bandwidth (to $\sim 1-10 \mathrm{~nm}$ ) and a decrease in the fluorescent lifetime from a few ns to hundreds of ps necessary for high-speed communication by using the properties of 1-D photonic bandgap structures in cholesteric LC, 2-D/3-D photonic crystals in polymerdispersed LCs, and photonic crystals/microstructured fibers infiltrated with LCs. Using various fluorescence emitters (dye molecules, colloidal semiconductor quantum dots, rods, carbon nanotubes and rare-earth ions) we will extend the working region of the source from the visible to communication wavelengths $(1.55 \mu \mathrm{m})$. 


\section{REFERENCES}

[1] Gisin, N., Ribordy, G., Tittel, W., \& Zbinden, H. (2002). Rev. Mod. Phys., 74, 145.

[2] Kwiat, P. G. (Ed.) (2002). Special Issue. Focus on quantum cryptography. New J. Phys., 4.

[3] Kumar, E. P., Kwiat, P., Migdall, A., Nam, S. W., Vuckovic, J., \& Wong, F. N. C. (2004). Quantum Information Processing, 3(1-5), 215.

[4] Grangier, P., Sanders, B., Vuckovic, J. (Eds.) (2004). Special issue. Focus on single photons on demand. New J. Phys., 6.

[5] Walls, D. F. \& Milburn, G. J. (1995). Quantum Optics, Springer Verlag: Berlin, NY.

[6] Benjamin, S. (2000). Science, 290, 2273.

[7] Knill, E., Laflamme, R., \& Milburn, G. J. (2001). Nature, 409, 46.

[8] (May 2003). Quantum cryptography: making it to market. Opt. \& Photonics News, 12.

[9] Yamamoto, Y., Santori, C., Solomon, G., Vuckovic, J., Fattal, D., Waks, E., Diamanti, E. (2005). Progress in Informatics, 1, 5.

[10] Ambrose, W. P., Goodwin, P. M., Enderlein, J., Semin, D. J., Martin, J. C., \& Keller, R. A. (1997). Chem. Phys. Lett., 269, 365.

[11] Lounis, B., Bechtel, H. A., Gerion, D., Alivisatos, P., \& Moerner, W. E. (2000). Chem. Phys. Lett., 329, 399.

[12] Kurtsiefer, C., Mayer, S., Zarda, P., \& Weinfurter, H. (2000). Phys. Rev. Lett., 85, 290.

[13] Lukishova, S. G., Schmid, A. W., McNamara, A. J., Boyd, R. W., \& Stroud, C. R. (2003). Spec. issue on Quantum internet technologies. IEEE J. Selected Topics in Quant. Electronics, 9(6), 1512.

[14] Lukishova, S. G., Schmid, A. W., Supranowitz, C. M., Lippa, N., McNamara, A. J., Boyd, R. W., \& Stroud, C. R. (2004). Special issue on Single photon: Detectors, applications and measurements methods. J. Mod. Optics, 51(9-10), 1535.

[15] Lukishova, S. G., Schmid, A. W., McNamara, A. J., Boyd, R. W., \& Stroud, C. R. (2003). LLE Review, Quarterly Report, DOE/SF/19460-485, Laboratory for Laser Energetics, University of Rochester, 94(Jan-March), 97.

[16] McKeever, J., Boca, A., Boozer, A. D., Miller, R., Buck, J. R., Kuzmich, A., \& Kimble, H. J. (2004). Science, 303, 1992.

[17] Lounis, B. \& Moerner, W. E. (2000). Nature, 407, 491.

[18] Vargas, F., Hollricher, O., Marti, O., De Schaetzen, G., \& Tarrach, G. (2002). J. Chem. Phys., 117, 866.

[19] Chen, H. M. P., Katsis, D., \& Chen, S. H. (2003). Chem. Mater., 15, 2534.

[20] Bunning, T. J. \& Kreuzer, F.-H. (1995). Trends in Polym. Sci., 3(10), 318.

[21] Schmidt, W. Institute for PAH Research, D-86926 Greifenberg am Ammersee, Germany.

[22] Hanbury Brown, R. \& Twiss, R. Q. (1956). Nature, 177, 27.

[23] Fleury, L., Segura, J.-M., Zumofen, G., Hecht, B., \& Wild, U. P. (2000). Phys. Rev. Lett., 84, 1148.

[24] Treussart, F., Clouqueur, A., Grossman, C., \& Roch, J.-F. (2001). Opt. Lett., 26, 1504

[25] Basché, Th., Morner, W. E., Orrit, M., \& Wild, U. P. (Eds.). (1997). Single Molecule Optical Detection, Imaging and Spectroscopy, VCH: Weinheim.

[26] Chung, I., Shimizu, K. T., \& Bawendi, M. G. (2003). PNAS, 100, 405.

[27] Stevens, B. \& Ha, T. (2004). J. Chem. Phys., 120, 3030.

[28] Axelrod, D. (1979). Biophys. J., 26, 557. 